\title{
Ameliorative effect of nicergoline on cognitive function through the PI3K/AKT signaling pathway in mouse models of Alzheimer's disease
}

\author{
GUOYAO ZANG $^{1}$, LIZHENG FANG ${ }^{1}$, LIYING CHEN $^{1}$ and CHENYAO WANG ${ }^{2}$ \\ ${ }^{1}$ Department of General Practice, Sir Run Run Shaw Hospital, School of Medicine, Zhejiang University, \\ Hangzhou, Zhejiang 310016; ${ }^{2}$ Department of Acupuncture and Moxibustion, The Third Affiliated \\ Hospital of Zhejiang Chinese Medical University, Hangzhou, Zhejiang 310005, P.R. China
}

Received August 28, 2016; Accepted September 26, 2017

DOI: $10.3892 / \mathrm{mmr} .2018 .8786$

\begin{abstract}
Alzheimer's disease is one of the most common age-associated diseases that frequently leads to memory disorders, cognitive decline and dementia. Evidence suggests that nicergoline serves an important role in the apoptosis of hippocampal cells, memory recovery, cognitive function and neuronal survival. However, the signaling pathway affected by nicergoline treatment remains to be elucidated. The purpose of the present study was to investigate the role of nicergoline in the cognitive competence of a mouse model of Alzheimer's disease. The apoptosis rates of hippocampal cells were studied in mice with Alzheimer's disease treated with nicergoline compared with the negative control. Apoptosis-associated gene expression levels in hippocampal cells, and hippocampus area, were analyzed in the experimental mice. Visual attention and inhibitory control were assessed and neural counting was performed in brain regions of interest. The phosphatidylinositol 3-kinase (PI3K)/R AC- $\alpha$ serine/threonine-protein kinase (AKT) signaling pathway was additionally analyzed in hippocampal cells following treatment with nicergoline. The results of the present study demonstrated that nicergoline ameliorated apoptosis in hippocampal cells and hippocampus tissue in 3xTg-AD mice with Alzheimer's disease. The data indicated that apoptosis-associated genes, including caspase-3, BCL2 associated X, BH3 interacting domain death agonist and caspase-9, were downregulated in hippocampal cells isolated from nicergoline-treated experimental mice. In addition, the expression levels of inflammatory factors, in
\end{abstract}

Correspondence to: Professor Chenyao Wang, Department of Acupuncture and Moxibustion, The Third Affiliated Hospital of Zhejiang Chinese Medical University, 219 Moganshan Road, Hangzhou, Zhejiang 310005, P.R. China

E-mail: wangchenyaohz@163.com

Key words: Alzheimer's disease, nicergoline, hippocampus cells, apoptosis, cognitive competence, phosphatidylinositol 3-kinase/RAC- $\alpha$ serine/threonine-protein kinase addition to oxidative stress, were decreased in hippocampal cells treated with nicergoline. Additionally, amyloid precursor protein accumulation was cleared in the hippocampal area in nicergoline-treated mice. Nicergoline inhibited neuronal loss and prevented cognitive impairment through the restoration of learning/memory ability. It was additionally demonstrated in the present study that nicergoline improved motor attention impairment and cognitive competence in hippocampal cells by acting on the PI3K/AKT signaling pathway. Therefore, memory recovery, cognitive function and neuronal survival were repaired by nicergoline via inhibition of the PI3K/AKT signaling pathway, suggesting that nicergoline may be an efficient drug for the clinical treatment of patients with Alzheimer's disease.

\section{Introduction}

Senile dementia is a multifactorial syndrome characterized by cognitive functional disorders and cognitive decline (1). Alzheimer's disease is among the most common pathogeneses leading to senile dementia, with a highest incidence rate among the elderly $(2,3)$. Alzheimer's disease is a progressive neurodegenerative syndrome characterized by the presence of neurodegenerative disorders (4). Alzheimer's disease primarily impairs hippocampus-associated memory and cognition, and leads to cognitive functional disorders and impairments in cognition, including memory, language or attention $(5,6)$. Previous studies have suggested that Alzheimer's may directly affect hippocampal cells (7-9). The association between corticocerebral and hippocampal neuronal apoptosis has been demonstrated in a rat model of Alzheimer's disease and in a clinical study $(10,11)$. Dysfunction and aberrant apoptosis of hippocampalcells has been observed in preclinical and clinical studies (12). Inhibition of neuronal apoptosis may attenuate the phosphorylation of microtubule-associated protein tau (MAPT) and protect memory, which may be beneficial for the treatment of Alzheimer's disease $(13,14)$. Absalon et al $(15)$ suggested that the activation of cell cycle entry, MAPT-phosphorylation and the inhibition of apoptosis in post-mitotic neurons through microRNA-regulated gene expression may contribute to the recovery of mice with Alzheimer's disease. These findings 
suggested that the apoptosis of hippocampal cells is associated with the progression of cognitive impairment, and thus with the onset and development of Alzheimer's disease.

The apoptosis of hippocampal cells is a diagnostic criterion for Alzheimer's disease $(16,17)$. Decreased apoptosis in the hippocampus signifies an improvement in cognitive impairment. Previous studies have supported the hypothesis that inflammatory responses are the most important pathogenic factors for the initiation and progression of Alzheimer's disease $(18,19)$. Although Alzheimer's disease has been extensively studied, comprehensive assessments have not been performed in an animal model. The majority of research in animal models of Alzheimer's disease has focused on the neuroprotective effects of anti-dementia drugs against inflammation, apoptosis and neuronal excitability (20). Oxidative stress has been associated with Alzheimer's disease and the development of neurodegenerative processes (21). Therefore, the development of pharmacological agents targeting apoptosis, inflammation and oxidative stress represents a novel prospect for the treatment of Alzheimer's disease.

Nicergoline is a derivative of ergot produced by semisynthesis that exhibits the potential to selectively block $\alpha-1 \mathrm{~A}$ adrenergic receptors in the brain and periphery (22). Nicergoline may improve a number of aging-associated diseases, including dysphagia, ischemia and dizziness (23-25). Nicergoline is beneficial for the treatment of cognitive impairment (26-28). At present, nicergolineis a registered drug in $>50$ countries and, for the last 30 years, it has been used for the treatment of cognitive impairment and behavioral disorders in the elderly. Therefore, nicergoline may contribute to the treatment of Alzheimer's disease.

In the present study, it was hypothesized that nicergoline may be able to protect neurons against apoptosis by controlling the expression of apoptosis-associated genes. The efficacy of nicergoline in the prevention of cognitive impairment was evaluated in a rat model of Alzheimer's disease and determined by the assessment of the learning memory. The molecular mechanism of nicergoline-mediated improvement in hippocampal cells was studied. Although nicergoline demonstrated neuroprotective effects via stimulation of the phosphatidylinositol 3-kinase (PI3K)/RAC- $\alpha$ serine/threonine-protein kinase (AKT) signaling pathway, further analysis of larger volumes of preclinical data is required to understand the potential efficacy and tolerability.

\section{Materials and methods}

Ethical approval. The present study was approved by the Ethics Committee of Zhejiang University (Hangzhou, China). All surgery and euthanasia of experimental mice was performed under sodium pentobarbital anesthesia to minimize suffering.

Cells and reagents. Hippocampal cells were isolated from 3xTg-AD mice and cultured in 1640 medium (Sigma-Aldrich; Merck KGaA, Darmstadt, Germany) supplemented with $10 \%$ fetal bovine serum (Gibco; Thermo Fisher Scientific, Inc., Waltham, MA, USA). Hippocampal cells were treated with PI3K inhibitor LY294002 (2 mg/ml) and cultured in for $12 \mathrm{~h} \mathrm{a}$ $37^{\circ} \mathrm{C}$ humidified atmosphere with $5 \% \mathrm{CO}_{2}$.
Animal study. A total of twenty 6-week-old 3xTg-AD mice (male, 28-35 g) with the Alzheimer's disease were purchased from Vital River Laboratory Animal Technology Co., Ltd. (Shanghai, China). All animals were housed in a temperature-controlled facility at $23 \pm 1^{\circ} \mathrm{C}$ and relative humidity of $50 \pm 5 \%$ with a 12 -h light/dark cycle with free access to food and water. The mice were divided into two group $(n=10$ in each group) and given intravenous injections of nicergoline (10 mg/kg body weight) or PBS (10 mg/kg body weight; both from Sigma-Aldrich; Merck KGaA) once a day. The treatment lasted 60 days.

Histologicalandimmunohistochemicalanalysis.Hippocampal tissues were isolated from the CA1 regions of experimental mice following a 60 -day treatment with nicergoline $(10 \mathrm{mg} / \mathrm{kg}$ body weight) or PBS. The brains were frozen in liquid nitrogen and following perfusion, fixation and cryoprotection, coronal sections were cut to $4 \mu \mathrm{m}$ in a cryostat. After blocking with 5\% Bull Serum Albumin (Sigma-Aldrich; Merck KGaA) for $1 \mathrm{~h}$ at $37^{\circ} \mathrm{C}$, free-floating sections were rinsed with PBS and placed in a solution with goat anti-mouse amyloid $\beta(A \beta)-42(1: 1,000$; cat. no. ab201060; Abcam, Cambridge, UK), A $\beta-40$ (1:1,000; cat. no. ab17295; Abcam) and amyloid precursor protein (APP; 1:1,000; cat. no. ab32136; Abcam) for $12 \mathrm{~h}$ at $4^{\circ} \mathrm{C}$. Following incubation the sections were washed and incubated with a secondary rabbit anti-goat antibody conjugated with horseradish peroxidase (1:500; cat. no. A-11034; Chemicon International; Thermo Fisher Scientific, Inc.) for $\mathrm{A} \beta-42$, $\mathrm{A} \beta-40$ and APP staining for $1 \mathrm{~h}$ at $37^{\circ} \mathrm{C}$. The sections were washed and observed using a fluorescent video microscope (BZ-9000; Keyence Corporation, Osaka, Japan) at magnification $\mathrm{x} 40$. Immunohistochemical staining was used to examine the abundance of neuroprotection-associated proteins in the hippocampus. Immunohistochemical procedures were performed as previously described (29).

Immunofluorescence. Hippocampal CA1 regions were isolated from experimental mice following treatment with nicergoline. Hippocampal neural cells were fixed in situ with bromodeoxyuridine and $\mathrm{NeuN}$ for $24 \mathrm{~h}$ at $4^{\circ} \mathrm{C}$. The impairment of neurogenesis was analyzed under a fluorescence microscope (Olympus BX61; Olympus Corporation, Tokyo, Japan) at magnification 40x.

Terminal deoxynucleotidyl transferase dUTP nick end labeling (TUNEL) assay. ATUNEL assay was performed to analyze apoptotic neurons in the hippocampus as previously described (30). Brain sections were viewed under alight microscope and of apoptotic morphology was labeled in sections. Magnification, $\mathrm{x} 40$.

ELISA analysis. ELISA kits [Huiying Chemical Industry (Xiamen) Co., Ltd., Shanghai, China] were used to determine interleukin (IL)-1 (cat. no. MLB00C; Bio-Rad Laboratories, Inc., Hercules, CA, USA), tumor necrosis factor (TNF)- $\alpha$ (cat. no. MTA00B; Bio-Rad Laboratories, Inc.) and IL-6 (cat. no. M6000B; Bio-Rad Laboratories, Inc.) serum levels in mice. The procedures were performed according to the manufacturer's protocol. The final results were recorded at a wavelength of $450 \mathrm{~nm}$. 
Table I. Sequences of primers used in this study.

Sequence

\begin{tabular}{lll}
\cline { 2 - 3 } Gene name & \multicolumn{1}{c}{ Reverse } & Forward \\
\hline P53 & 5'-TTAAGCTTTTTGCGTTCGGGCTGGGAGC-3' & 5'-A TGGTGGCATGAACCTGTGG-3' \\
Bcl-2 & 5'-CGTCATAACTAAAGACACCCC-3' & 5'-TTCATCTCCAGTATCCGACTC-3' \\
Caspase-3 & 5'-ATGGAGAACAACAAAACCTCAGT-3' & 5'-TTGCTCCCATGTATGGTCTTTAC-3' \\
Caspase-9 & 5'-GCCCTTGCCTCTGAGTAGTG-3' & 5'-CCAACCAAATGAAGCCAAGT-3' \\
Bax & 5'-CACCAATCACCTGCGGTACA-3' & 5'-CAGATCACGTCATCGCAC-3' \\
Bid & 5'-CGACGAGGTGAAGACATCCT-3' & 3'-AGCAGAGATGGTGCATGACT-3' \\
-actin & 5'-CCTTCCTGGGCATGGAGTCCT-3' & 5'-GGAGCAATGATCTTGATCTTC-3'
\end{tabular}

Bcl-2, B-cell lymphoma-2; Bax, B-cell lymphoma-2 associated X-protein; Bid, BH3 interacting-domain death agonist.

Flow cytometry. The apoptosis of hippocampal neural cells was evaluated using an Annexin V-fluorescein isothiocyanate (FITC) Apoptosis Staining/Detection kit (cat. no. ab14085; Abcam, Cambridge, UK) and a propidium iodide (PI) apoptosis detection kit (BD Biosciences, Franklin Lakes, NJ, USA). Hippocampal neural cells were isolated from experimental mice and suspended with Annexin V-FITC and PI, according to the manufacturer's protocols. Fluorescence was measured via fluorescence-activated cell sorting using a flow cytometer (BD Biosciences). Cell apoptosis was analyzed using Expo32-ADC v. 1.2B software (Beckman Coulter, Inc., Brea, CA, USA).

Fluorescent reverse transcription-quantitative polymerase chain reaction ( $R T-q P C R)$ analysis. Total RNA was extracted from experimental mouse hippocampal are as using RNA Easy Mini Extract kit (Sigma-Aldrich; Merck KGaA) and the RNA served as a template for cDNA synthesis by RT reaction using a PrimeScript RT Master mix kit (Takara Biotechnology Co., Ltd., Dalian, China). A total of one-tenth of the cDNA was used in the fluorescent RT-qPCR reaction using iQ SYBR-Green (Invitrogen; Thermo Fisher Scientific, Inc.). The PCR conditions included an initial denaturation step of $94^{\circ} \mathrm{C}$ for $2 \mathrm{~min}$, followed by 30 cycles of $94^{\circ} \mathrm{C}$ for $30 \mathrm{sec}, 59^{\circ} \mathrm{C}$ for $30 \mathrm{sec}, 72^{\circ} \mathrm{C}$ for $2 \mathrm{~min}$ and a final elongation step at $72^{\circ} \mathrm{C}$ for $10 \mathrm{~min}$. All forward and reverse primers were summarized in Table I. Relative mRNA expression was calculated by the $2^{-\Delta \Delta \mathrm{Cq}}$ calculation (31). Results were presented as a fold difference relative to the housekeeping $\beta$-actin control gene.

Western blotting. Hippocampal cells were homogenized in radioimmunoprecipitation assay lysis buffer containing protease inhibitor (Sigma-Aldrich; Merck KGaA) and centrifuged at $6,000 \mathrm{x} \mathrm{g}$ at $4^{\circ} \mathrm{C}$ for $10 \mathrm{~min}$. The supernatant was used for the analysis of target proteins using a protein extraction kit (Qiagen Sciences, Inc., Gaithersburg, MD, USA), according to manufacturer's protocol. The protein concentration was measured in samples by a bicinchoninic acid protein assay kit (Thermo Fisher Scientific, Inc.). Protein samples $(10 \mu \mathrm{g})$ were separated on $12.5 \%$ SDS-PAGE gels and transferred onto polyvinylidene difluoride membranes (EMD Millipore, Billerica, MA, USA). SDS-PAGE was performed as previously described (32). For western blotting, primary antibodies: IL-1 $\beta$ (1:400; cat. no. ab200478), IL-6, TNF- $\alpha$ (1:500; cat. no. ab1793), Foxp2 (1:400; cat. no. ab16046), Src homology 2-containing inositol phosphatase (SxIP; 1:400; cat. no. ab45142), EB (1:400; cat. no. ab157217), P53 (1:400; cat. no. ab26), Bcl-2 (1:400; cat. no. ab32124), caspase-3 (1:500; cat. no. ab217), caspase-9 (1:500; cat. no. ab52298), Bid (1:400; cat. no. ab62469), Bax (1:400; cat. no. ab32124), Neprilysin (1:500; cat. no. ab79423), insulin (1:500; cat. no. ab32216), insulin-like growth factor-binding protein 3 (IGFBP-3; 1:500; cat. no. ab75988), vascular endothelial growth factor $\beta$ (VEGF- $\beta$; $1: 500$; cat. no. ab53463), superoxide dismutase (SOD; 1:500; cat. no. ab13533), catalase (CAT; 1:500; cat. no. ab16731), glutathione (GSH; 1:500; cat. no. ab26255), PI3K (1:1,000; cat. no. ab182651; Thermo Fisher Scientific, Inc.), AKT (1:1,000; cat. no. ab8933; Thermo Fisher Scientific, Inc.), pAKT (1:1,000; cat. no. ab18260; Thermo Fisher Scientific, Inc.), ROS (1:1,000; cat. no. PA5-14732; Thermo Fisher Scientific, Inc.) and $\beta$-actin (1:2,000, cat. no. ab8226) were added following blocking with 5\% skimmed milk for $1 \mathrm{~h}$ at $37^{\circ} \mathrm{C}$, then the membranes incubated with primary antibodies overnight at $4^{\circ} \mathrm{C}$ and then incubated with secondary antibodies (1:5,000; PV-6001; OriGene Technologies, Inc., Rockville, $\mathrm{MD}, \mathrm{USA}$ )for $1 \mathrm{~h}$ at $37^{\circ} \mathrm{C}$. Finally, protein bands were visualized using WesternBright Enhanced Chemiluminescent substrate (Advansta, Inc., Menlo Park, CA, USA).

Cognitive and behavioral tests. The cognitive competence of mice was determined by measuring the open field activity levels of mice in black plexiglass boxes $(60 \times 60 \times 25 \mathrm{~cm})$, to analyze the therapeutic effects of nicergoline. Mice were placed in open black boxes for $10 \mathrm{~min}$ and the behavior of the mice was monitored and evaluated using an auto-tracking system (EthoVision 8.0; Noldus, Wageningen, the Netherlands). A Morris water maze test was performed prior to and post-treatment with nicergoline to measure cognitive function. The Morris water maze experiment was performed three times in a circular stainless steel tank (155 cm diameter, $60 \mathrm{~cm}$ depth) filled with water to a depth of $40 \mathrm{~cm}\left(27.0 \pm 1.0^{\circ} \mathrm{C}\right)$ that was made opaque by the addition of skimmed milk. Mice learned to find a hidden circular platform (10 $\mathrm{cm}$ diameter, $1 \mathrm{~cm}$ below the surface of the water) in a fixed area in one quadrant of the tank. 
A

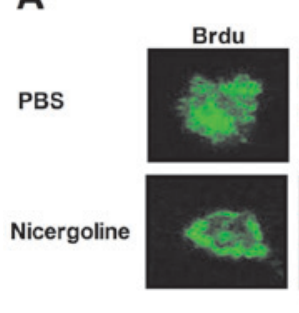

D

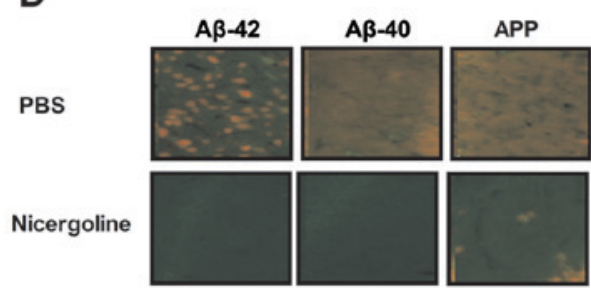

B
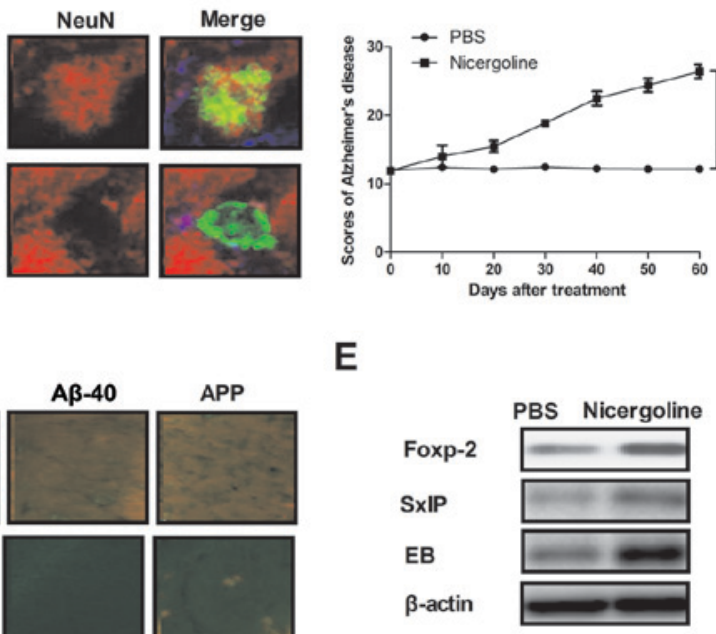

C

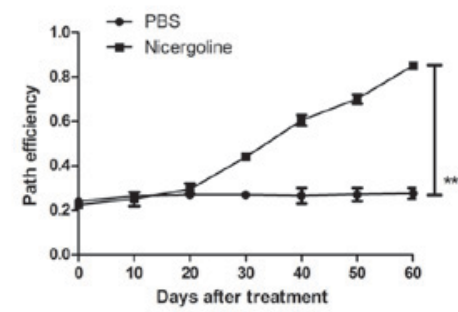

$\mathbf{F}$

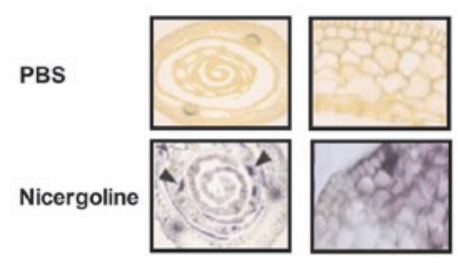

Figure 1. In vivo efficacy of nicergoline for impaired neurogenesis and cognitive competence in mice with Alzheimer's disease. (A) Analysis of impaired neurogenesis determined by immunofluorescence in mice treated with nicergoline. (B) Evaluation of cognitive competence of mice with Alzheimer's disease treated with nicergoline. (C) Degree of dementia determined by path efficiency in mice receiving treatment with nicergoline compared with the control. (D) Abundance of the pathogenic factors A $\beta-42, A \beta-40$ and APP in the hippocampus of experimental mice, determined by immunohistochemistry. (E) Levels of neuroprotective proteins Foxp-2, SxIP and EB in hippocampus of nicergoline-treated mice determined by western blotting. (F) Hippocampus excitability following treatment with nicergoline compared with the control group. Arrowheads indicate the dispersion of the pyramidal cell layer. All data are presented as the mean \pm standard error of the mean. ${ }^{* *} \mathrm{P}<0.01$ vs. PBS. APP, amyloid precursor protein; Foxp-2, forkhead box protein P2; A $\beta$, amyloid $\beta$; APP, amyloid precursor protein; EB, end-binding proteins; SxIP, Src homology 2-containing inositol phosphatase; Brdu, bromodeoxyuridine.

Thionin staining. Brain sections (30 $\mu \mathrm{m}$ thick) were prepared and stained with thionin for $2 \mathrm{~h}$ at room temperature. Images of stained tissues were digitally captured with an Olympus IX71 microscope controlled by DP manager software (Olympus Corporation, Tokyo, Japan) at a magnification of x1.5.

Statistical analyses. All data are presented as the mean \pm standard error of the mean. Statistical significance was determined using a two-tailed Student's t-test with SPSS 19.0 (IBM Corp., Armonk, NY, USA). One-way analysis of variance followed by Tukey's post hoc test, Kaplan-Meier analysis and log-rank statistical analysis were performed using GraphPad software version 5.0 (GraphPad Software, Inc., La Jolla, CA, USA). $\mathrm{P}<0.05$ was considered to indicate a statistically significant difference.

\section{Results}

Nicergoline improves impaired neurogenesis and cognitive competence in mice with Alzheimer's disease. In order to analyze the efficacy of nicergolinein the treatment of Alzheimer's disease, mice with Alzheimer's disease received treatment with nicergoline $(10 \mathrm{mg} / \mathrm{kg}$ body weight $)$ or were administered PBS as a control. It was determined that nicergoline treatment markedly improved neurogenesis and cognitive competence compared with the control (Fig. 1A and $\mathrm{B})$. In mice that received treatment with nicergoline, the degree of dementia was decreased following a 60-day course of treatment (Fig. 1C). Immunological staining indicated that pathogenic A $\beta-42$ and -40 peptides and APP were downregulated in the hippocampi of experimental mice (Fig. 1D). Levels of the neuroprotective forkhead box protein P2 (Foxp2), Src homology 2-containing inositol phosphatase (SxIP) and end-binding proteins (EB) were increased in hippocampi of nicergoline-treated mice (Fig. 1E). Additionally, marked differences in the dispersion of the pyramidal cell layer were observed between the nicergoline-treated and control groups, as determined by thionin staining (Fig. 1F). These results suggested that nicergoline exerted beneficial effects on neurogenesis and cognitive competence in mice with Alzheimer's disease.

Nicergoline inhibits apoptosis in hippocampal cells from mice with Alzheimer's disease. As previously indicated, apoptosis of hippocampal cells is ubiquitous in patients with Alzheimer's disease (33). Therefore, the present study examined the apoptosis of hippocampal cells in a mouse model of Alzheimer's disease following treatment with nicergoline or PBS. It was demonstrated that nicergoline inhibited apoptosis in hippocampal cells isolated from mice with Alzheimer's disease and analyzed by flow cytometry in vitro (Fig. 2A). The TUNEL assay demonstrated that apoptotic hippocampal cells were significantly less prevalent in nicergoline-treated mice in vivo (Fig. 2B). In addition, the expression levels of apoptotic factors in hippocampal cells isolated from nicergoline-treated mice were investigated. The mRNA and protein levels of pro-apoptotic caspase-3, BCL2 associated X (Bax), BH3 interacting domain death agonist (Bid) and caspase-9 were downregulated in the hippocampal cells of nicergoline-treated mice (Fig. 2C and D). However, the mRNA and protein levels of anti-apoptotic p53 and apoptosis regulator Bcl-2 were upregulated in the hippocampal cells of nicergoline-treated mice (Fig. 2E and F). The results of the present study indicated that nicergoline may inhibit apoptosis in hippocampal cells by regulating the activation of apoptosis-associated genes.

Nicergoline inhibits inflammation and oxidative stress in hippocampal cells from mice with Alzheimer's disease. The 
A

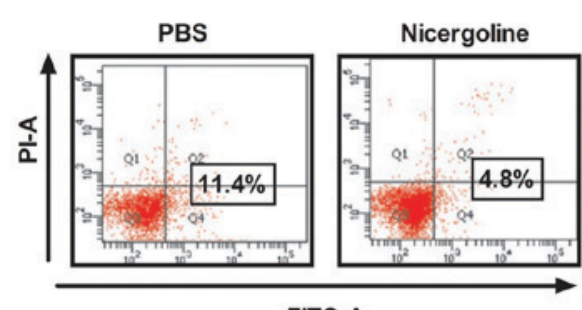

FITC-A

D

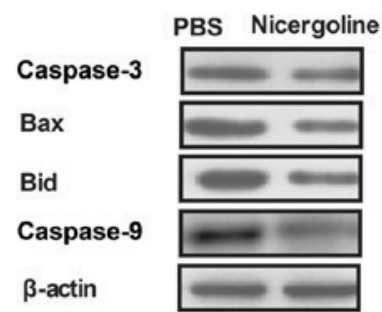

B

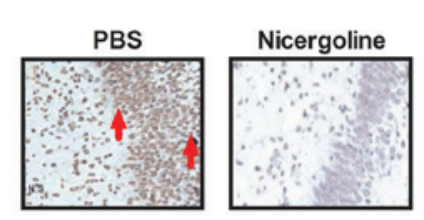

E

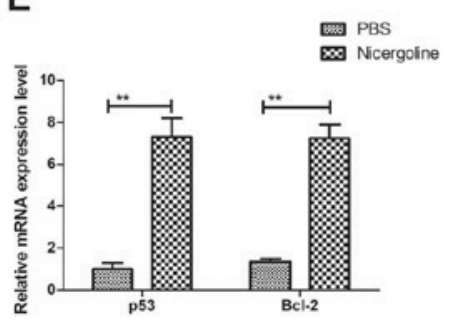

C

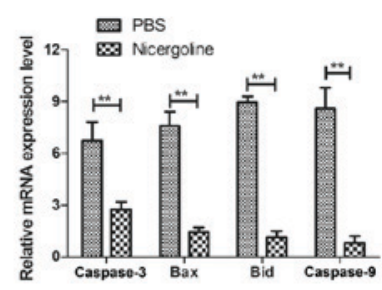

F

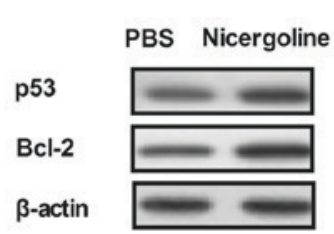

Figure 2. Analysis of apoptosis in hippocampal cells from mice with Alzheimer's disease. (A) The apoptosis of hippocampal cells isolated from mice with Alzheimer's disease was analyzed by flow cytometry in vitro. (B) Terminal deoxynucleotidyl transferase dUTP nick end labeling assay of hippocampal cells in nicergoline-treated mice in vivo. Arrowheads indicate the apoptotic hippocampal cells. (C) Pro-apoptotic mRNA expression levels in hippocampal cells isolated from nicergoline-treated mice. (D) Protein levels of caspase-3, Bax, Bid and caspase-9 in hippocampal cells isolated from nicergoline-treated mice. (E) Anti-apoptotic mRNA in hippocampal cells isolated from nicergoline-treated mice. (F) Protein levels of p53 and Bcl-2 in hippocampal cells isolated from nicergoline-treated mice. All data are presented as the mean \pm standard error of the mean. ${ }^{* * *} \mathrm{P}<0.01$ vs. PBS. Bax, BCL2 associated X; Bid, BH3 interacting domain death agonist; Bcl-2, apoptosis regulator Bcl-2; FITC, fluorescein isothiocyanate; PI, propidium iodide.

A

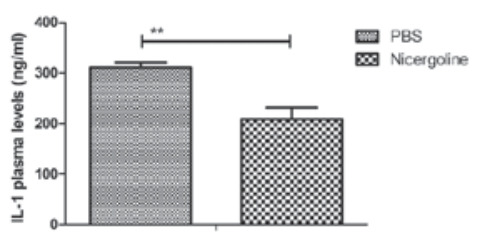

D
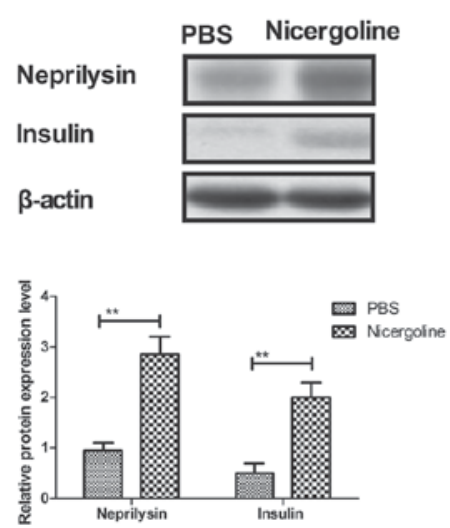

B

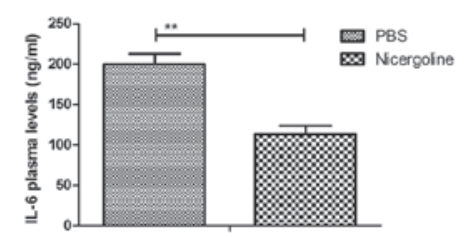

E
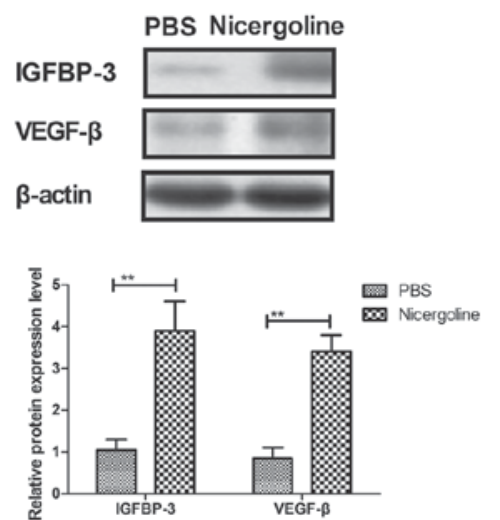

C

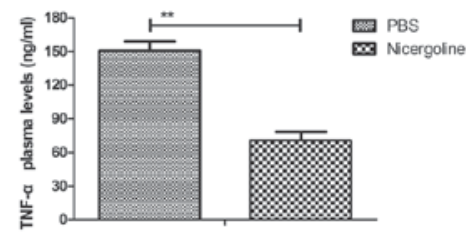

$\mathbf{F}$
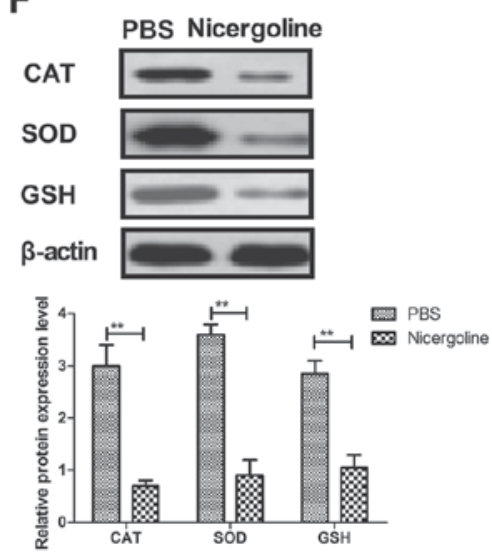

Figure 3. Analysis of inflammation and oxidative stress in hippocampal cells from mice with Alzheimer's disease. (A) Plasma concentration of IL-1 in hippocampal cells treated with nicergoline as determined by ELISA analysis. (B) Plasma concentration of IL-6 in hippocampal cells treated with nicergoline as determined by ELISA analysis. (C) Plasma concentration of TNF- $\alpha$ in hippocampal cells treated with nicergoline as determined by ELISA analysis. (D) Neprilysin and insulin expression levels in hippocampal cells treated with nicergoline as determined by western blotting. (E) Expression levels of IGFBP-3 and VEGF- $\beta$ in hippocampal cells treated with nicergoline. (F) Expression levels of ROS, SOD and GSH in in hippocampal cells treated with nicergoline. All data are presented as the mean \pm standard error of the mean. ${ }^{* * *} \mathrm{P}<0.01$ vs. PBS. IL, interleukin; IGFBP-3, insulin-like growth factor-binding protein 3 ; VEGF- $\beta$, vascular endothelial growth factor $\beta$; ROS, reactive oxygen species; SOD, superoxide dismutase; GSH, glutathione; TNF- $\alpha$, tumor necrosis factor- $\alpha$.

expression levels of inflammatory factors in hippocampal cells from experimental mice were examined. It was demonstrated that the plasma concentrations of IL-1, IL- 6 and TNF- $\alpha$ were decreased in hippocampal cells treated with nicergoline
(Fig. 3A-C). Neprilysin and insulin expression levels were upregulated in hippocampal cells in the mice with Alzheimer's disease treated with nicergoline for 60 days (Fig. 3D). In addition, insulin-like growth factor-binding protein 3 and vascular 
A
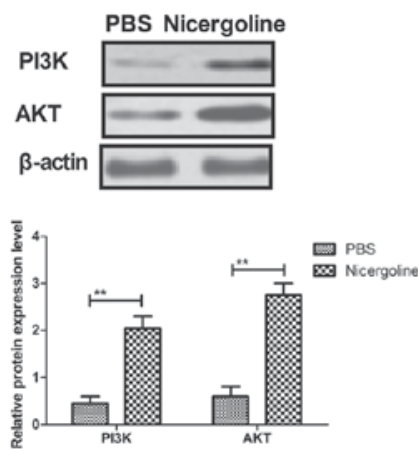

D
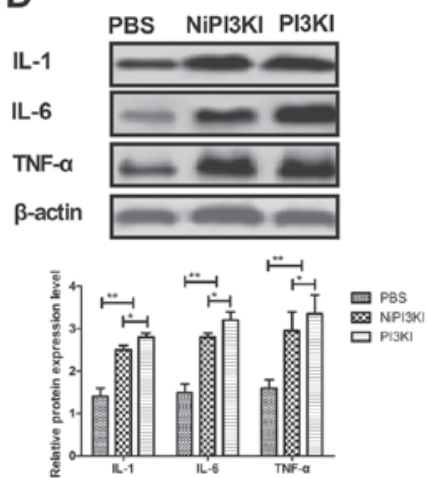

B
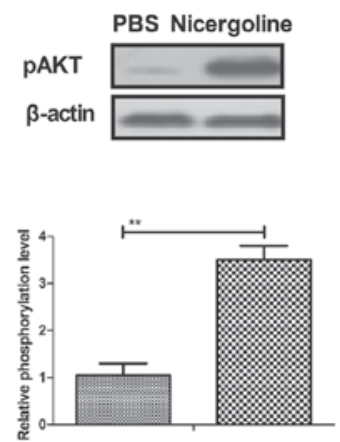

E

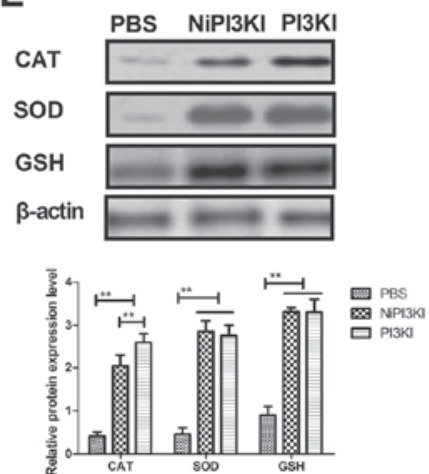

C
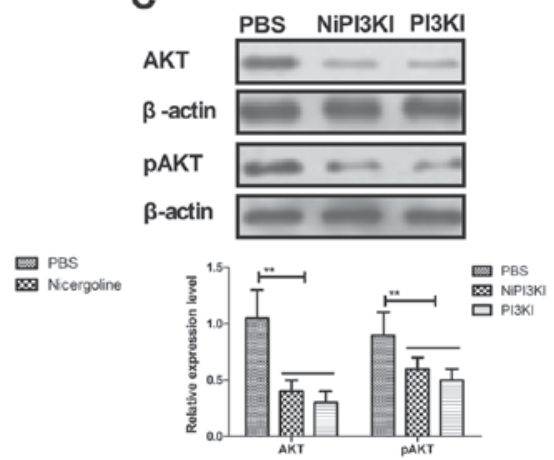

$\mathbf{F}$

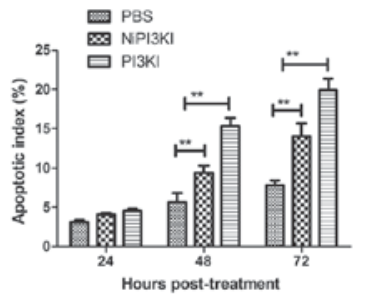

Figure 4. Nicergoline-regulated activity of hippocampal cells through the PI3K/AKT signaling pathway. (A) The effect of treatment with nicergoline on the protein expression of PI3K and AKT in hippocampal cells isolated from experimental mice. (B) The phosphorylation of AKT in the hippocampus in nicergoline-treated mice with Alzheimer's disease in vivo. (C) The effect of inhibition of PI3K activity on AKT expression and phosphorylation. (D) The effect of inhibition of PI3K activity on the expression of inflammatory factors in hippocampal cells in vitro. (E) The effect of inhibition of PI3K activity on oxidative stress in the hippocampus in vitro. (F) The effect of inhibition of PI3K activity on the apoptosis rate in hippocampal cells in vitro. All data are presented as the mean \pm standard error of the mean. ${ }^{*} \mathrm{P}<0.05,{ }^{* *} \mathrm{P}<0.01$ vs. PBS. PI3K, phosphatidylinositol 3-kinase; AKT, RAC- $\alpha$ serine/threonine-protein kinase; PI3KI, PI3K inhibitor; NiPI3KI, nicergoline + PI3KI; IL, interleukin; TNF- $\alpha$, tumor necrosis factor- $\alpha$; CAT, catalase; SOD, superoxide dismutase; GSH, glutathione.

endothelial growth factor $\beta$ protein levels were increased in hippocampal cells in the experimental mice treated with nicergoline (Fig. 3E). Oxidative stress differences between experimental mice we analyzed following treatment with nicergoline. As presented in Fig. 3F, the expression levels of reactive oxygen species, superoxide dismutase and glutathione were downregulated in hippocampal cells in the experimental mice treated with nicergoline. The results of the present study indicated that nicergoline mitigated Alzheimer's disease in mice via inhibition of inflammation and oxidative stress in hippocampal cells.

Nicergoline regulates the activity of hippocampal cells through the PIBK/AKT signaling pathway. In order to elucidate the nicergoline-mediated mitigation of Alzheimer's disease in mice, molecular alterations in the PI3K/AKT signaling pathway in hippocampal cells were studied. As presented in Fig. 4A, treatment with nicergoline significantly increased the expression of PI3K and AKT in hippocampal cells isolated from experimental mice. In addition, nicergoline significantly promoted the phosphorylation of AKT compared with the negative control (Fig. 4B). It was observed that inhibition of PI3K activity (PI3KI) by LY294002 obviated the AKT expression and phosphorylation stimulated by treatment with nicergoline (Nicergoline + PI3KI; NiPI3KI) in hippocampal cells (Fig. 4C). In addition, the expression levels of IL-1, IL-6 and TNF- $\alpha$ were upregulated in PI3K-inhibitedhippocampal cells in vitro (Fig. 4D). Additionally, oxidative stress was increased in PI3K-inhibited hippocampal cells in vitro (Fig. 4E). Notably, the apoptosis rate was increased in PI3K inhibitor-treated hippocampal cells in vitro (Fig. 4F). The aforementioned results demonstrated that nicergoline regulated the activity of hippocampal cells through stimulation of the PI3K/AKT signaling pathway.

\section{Discussion}

In the present study, the effect of nicergoline on Alzheimer's disease and the mechanism of action of nicergoline were analyzed. The results of the present study contributed to the efforts to develop an effective treatment for Alzheimer's disease. Nicergoline is as an efficient drug for the treatment of the cranial nerve symptoms associated with dementia, although there is no available data on its molecular mechanism of action in Alzheimer's disease. The PI3K/AKT pathway has been hypothesized to mediate the nicergoline-induced therapeutic effect on Alzheimer's disease in a mouse model. The results of the present study confirmed that nicergoline regulated PI3K/AKT expression and phosphorylation in the hippocampus of mice with Alzheimer's disease. The findings of the present study indicated that nicergoline inhibited inflammation, apoptosis and oxidative stress through upregulation of the PI3K/AKT signaling pathway.

The PI3K/AKT signaling pathway serves an essential role in cell growth, proliferation and survival under 
physiological conditions (34). There is scarce evidence of the association between the PI3K/AKT signaling pathway and Alzheimer's disease. Kong et al (35) reported that nicorandil serves a neuroprotective role in a cellular model of Alzheimer's disease through activation of the PI3K/AKT signaling pathway. Kitagishi et al (36) demonstrated that the $\mathrm{PI} 3 \mathrm{~K} / \mathrm{AKT} / \mathrm{glycogen}$ synthase kinase-3 $\beta$ pathway is involved in cell signaling in neuronal cells affected by Alzheimer's disease. In addition, ginseng induced a neuroprotective effect on D-galactose/aluminum chloride via the PI3K/AKT signaling pathway in the hippocampus, improved memory and reduced the content of A $\beta-42$ and MAPT in rats with Alzheimer's disease (37). Additionally, following treatment with salidroside, the PI3K/AKT signaling pathway ameliorated toxicity in A $\beta$-treated primary brain neural cultures (38). These previous reports suggested that neuroprotection is associated with the activity of the PI3K/AKT signaling pathway in hippocampal cells in cellular and animals models of Alzheimer's disease. The results of the present study indicated that nicergoline was able to improve neurogenesis and cognitive competence through upregulation of the PI3K/AKT signaling pathway and inhibition of inflammation, apoptosis and oxidative stress in mice with Alzheimer's disease.

Alzheimer's disease is characterized by apoptosis of hippocampal cells, senile plaques, oxidative stress, neurofibrillary tangles and inflammation (39). Chronic inflammatory responses stimulate the progression of Alzheimer's disease (40). Quinn et al (41) studied the association between inflammation and cerebral amyloidosis in an animal model of Alzheimer's disease. Balez et al (20) reported that apigenin may induce neuroprotective effects in a pluripotent stem cell model of Alzheimer's disease via inhibition of inflammation, improvement of neuronal excitability and reduced apoptosis. The results of the present study demonstrated that nicergoline was able to reduce inflammation via downregulation of inflammatory factors in hippocampal cells. Downregulation of inflammation contributes to reduced apoptosis in hippocampal cells. In addition, chronic inflammatory responses and oxidative stress are associated with the expression of $A \beta$ protein during the progression of Alzheimer's disease (42). The results of the present study suggested that nicergoline was able to improve $A \beta$ protein accumulation in the hippocampi of experimental mice. Additionally, apoptosis, a ubiquitous biological phenomenon in cells, is genetically controlled. Bcl-2 and caspase-3 are two key molecular apoptosis regulators and their function in the regulation of brain cell apoptosis was previously reported (43-46). One study demonstrated that downregulation of $\mathrm{Bcl}-2$ and simultaneous upregulation of Bax and Bid activated p53 and caspase-3, and consequently induced neuronal apoptosis in the rat hippocampus (47). Bcl-2 and caspase- 3 are two important molecular regulators of apoptosis and overexpression of $\mathrm{Bcl}-2$ inhibited the activation of caspase-3 and apoptosis (48). Therefore, the data presented in the present study may contribute to the understanding of the therapeutic properties of nicergoline for the treatment of Alzheimer's disease.

In conclusion, the results of the present preclinical study suggested that nicergoline may be an effective drug for the treatment of impaired neurogenesis and cognitive competence in Alzheimer's disease. Treatment with nicergoline markedly improved visual attention, inhibitory control and the neural cell count via inhibition of apoptosis in hippocampal cells and prevention of the expression of inflammatory mediators in mice with Alzheimer's disease. Notably, nicergoline-mediated activation of the PI3K/AKT signaling pathway was investigated in mice with Alzheimer's disease. The results suggested that nicergoline regulated neurogenesis and cognitive competence through the PI3K/AKT signaling pathway in the hippocampal cells of experimental mice. In conclusion, the results of the present study suggested that nicergoline may be a promising drug for the treatment of Alzheimer's disease.

\section{References}

1. Armstrong RA: Survival in the pre-senile dementia frontotemporal lobar degeneration with TDP-43 proteinopathy: Effects of genetic, demographic and neuropathological variables. Folia Neuropathol 54: 137-148, 2016.

2. Bando $\mathrm{N}$ and Nakamura $\mathrm{Y}$ : Preliminary evidence that rivastigmine-induced inhibition of serum butyrylcholinesterase activity improves behavioral symptoms in Japanese patients with Alzheimer's disease. Geriatr Gerontol Int 17: 1306-1312, 2017.

3. Verkhratsky A, Rodriguez-Arellano JJ, Parpura V and Zorec R: Astroglial calcium signalling in Alzheimer's disease. Biochem Biophys Res Commun 483: 1005-1012, 2017.

4. Cortese GP and Burger C: Neuroinflammatory challenges compromise neuronal function in the aging brain: Postoperative cognitive delirium and Alzheimer's disease. Behav Brain Res 322: 269-279, 2017.

5. Pettigrew C, Soldan A, Zhu Y, Wang MC, Brown T, Miller M and Albert M; BIOCARD Research Team: Cognitive reserve and cortical thickness in preclinical Alzheimer's disease. Brain Imaging Behav 11: 357-367, 2017.

6. Fritz NE, Kegelmeyer DA, Kloos AD, Linder S, Park A, Kataki M, Adeli A, Agrawal P, Scharre DW and Kostyk SK: Motor performance differentiates individuals with Lewy body dementia, Parkinson's and Alzheimer's disease. Gait Posture 50: 1-7, 2016.

7. Stella F, Laks J, Govone JS, de Medeiros K and Forlenza OV: Association of neuropsychiatric syndromes with global clinical deterioration in Alzheimer's disease patients. Int Psychogeriatr 28: 779-786, 2016.

8. Garcia KO, Ornellas FL, Martin PK, Patti CL, Mello LE, Frussa-Filho R, Han SW and Longo BM: Therapeutic effects of the transplantation of VEGF overexpressing bone marrow mesenchymal stem cells in the hippocampus of murine model of Alzheimer's disease. Front Aging Neurosci 6: 30, 2014.

9. Ramos B, Baglietto-Vargas D, del Rio JC, Moreno-Gonzalez I, Santa-Maria C, Jimenez S, Caballero C, Lopez-Tellez JF, Khan ZU, Ruano D, et al: Early neuropathology of somatostatin/NPY GABAergic cells in the hippocampus of a PS1xAPP transgenic model of Alzheimer's disease. Neurobiol Aging 27: 1658-1672, 2006.

10. Youn H, Ji I, Ji HP, Markesbery WR and Ji TH: Under-expression of Kalirin-7 Increases iNOS activity in cultured cells and correlates to elevated iNOS activity in Alzheimer's disease hippocampus. J Alzheimers Dis 12: 271-281, 2007.

11. Namba T, Maekawa M, Yuasa S, Kohsaka S and Uchino S: The Alzheimer's disease drug memantine increases the number of radial glia-like progenitor cells in adult hippocampus. Glia 57: 1082-1090, 2009.

12. Chen J, Shu H, Wang Z, Liu D, Shi Y, Xu L and Zhang Z: Protective effect of APOE epsilon 2 on intrinsic functional connectivity of the entorhinal cortex is associated with better episodic memory in elderly individuals with risk factors for Alzheimer's disease. Oncotarget 7: 58789-58801, 2016.

13. Ando K, Oka M, Ohtake Y, Hayashishita M, Shimizu S, Hisanaga S and Iijima KM: Tau phosphorylation at Alzheimer's disease-related Ser356 contributes to tau stabilization when PAR-1/MARK activity is elevated. Biochem Biophys Res Commun 478: 929-934, 2016.

14. Liu W, Zhao J and Lu G: miR-106b inhibits tau phosphorylation at Tyr18 by targeting Fyn in a model of Alzheimer's disease. Biochem Biophys Res Commun 478: 852-857, 2016. 
15. Absalon S, Kochanek DM, Raghavan V and Krichevsky AM: MiR-26b, upregulated in Alzheimer's disease, activates cell cycle entry, tau-phosphorylation, and apoptosis in postmitotic neurons. J Neurosci 33: 14645-14659, 2013.

16. Jahng GH, Oh J, Lee DW, Kim HG, Rhee HY, Shin W, Paik JW, Lee KM, Park S, Choe BY and Ryu CW: Glutamine and glutamate complex, as measured by functional magnetic resonance spectroscopy, alters during face-name association task in patients with mild cognitive impairment and Alzheimer's disease. J Alzheimers Dis 53: 745, 2016.

17. Nesteruk T,Nesteruk M,StyczynskaM,Barcikowska-Kotowicz M and Walecki J: Radiological evaluation of strategic structures in patients with mild cognitive impairment and early Alzheimer's disease. Pol J Radiol 81: 288-294, 2016.

18. Minter MR, Zhang C, Leone V, Ringus DL, Zhang X, Oyler-Castrillo P, Musch MW, Liao F, Ward JF, Holtzman DM, et al: Antibiotic-induced perturbations in gut microbial diversity influences neuro-inflammation and amyloidosis in a murine model of Alzheimer's disease. Sci Rep 6: 30028, 2016.

19. Huang Y, Zhao Z, Wei X, Zheng Y, Yu J, Zheng J and Wang L: Long-term trihexyphenidyl exposure alters neuroimmune response and inflammation in aging rat: Relevance to age and Alzheimer's disease. J Neuroinflammation 13: 175, 2016.

20. Balez R, Steiner N, Engel M, Muñoz SS, Lum JS, Wu Y, Wang D, Vallotton P, Sachdev P, O'Connor M, et al: Neuroprotective effects of apigenin against inflammation, neuronal excitability and apoptosis in an induced pluripotent stem cell model of Alzheimer's disease. Sci Rep 6: 31450, 2016

21. Justin Thenmozhi A, William Raja TR, Manivasagam T, Janakiraman U and Essa M: Hesperidin ameliorates cognitive dysfunction, oxidative stress and apoptosis against aluminium chloride induced rat model of Alzheimer's disease. Nutr Neurosci 20: 360-368, 2017.

22. Kim SY, Yang J and Lee YC: The effects of nicergoline on corneal nerve regeneration in rat corneas after photorefractive keratectomy. Curr Eye Res 36: 29-33, 2011.

23. Nakashima T, Hattori N, Okimoto M, Yanagida J and Kohno N: Nicergoline improves dysphagia by upregulating substance $\mathrm{P}$ in the elderly. Medicine 90: 279-283, 2011

24. Pilkowska E, Jakubowska T, Witkowska K and Kulczycki J: Nicergoline in the treatment of patients after a mild ischemic stroke. Neurol Neurochir Pol 36: 1075-1085, 2002 (In Polish).

25. Felisati G, Pignataro O, Di Girolamo A, Bruno E, Alessandrini M, Guidetti G, Monzani D, Beldi AM, Mira E, Benazzo M, et al Nicergoline in the treatment of dizziness in elderly patients. A review. Arch Gerontol Geriatr Suppl: 163-170, 2004

26. Saletu B, Garg A and Shoeb A: Safety of nicergoline as an agent for management of cognitive function disorders. Biomed Res Int 2014: 610103, 2014.

27. Caraci F, Chisari M, Frasca G, Canonico PL, Battaglia A, Calafiore M, Battaglia G, Bosco P, Nicoletti F, Copani A and Sortino MA: Nicergoline, a drug used for age-dependent cognitive impairment, protects cultured neurons against beta-amyloid toxicity. Brain Res 1047: 30-37, 2005

28. Fioravanti M and Flicker L: Efficacy of nicergoline in dementia and other age associated forms of cognitive impairment. Cochrane Database Syst Rev: CD003159, 2001.

29. Dirani M, Nasreddine W, Abdulla F and Beydoun A: Seizure control and improvement of neurological dysfunction in Lafora disease with perampanel. Epilepsy Behav Case Rep 2: 164-166, 2014.

30. Sharma R, Ahmad G, Esteves SC and Agarwal A: Terminal deoxynucleotidyl transferase dUTP nick end labeling (TUNEL) assay using bench top flow cytometer for evaluation of sperm DNA fragmentation in fertility laboratories: Protocol, reference values, and quality control. J Assist Reprod Genet 33: 291-300, 2016.

31. Livak KJ and Schmittgen TD: Analysis of relative gene expression data using real-time quantitative PCR and the 2(-Delta Delta C(T)) method. Methods 25: 402-408, 2001
32. Wai-Hoe L, Wing-Seng L, Ismail Z and Lay-Harn G: SDS-PAGE-based quantitative assay for screening of kidney stone disease. Biol Proced Online 11: 145-160, 2009.

33. Thinnes FP: Apoptogenic interactions of plasmalemmal type-1 VDAC and Abeta peptides via GxxxG motifs induce Alzheimer's disease-a basic model of apoptosis? Wien Med Wochenschr 161: 274-276, 2011.

34. Shi LX, Wang JH and Shi XD: PI3K/AKT/mTOR pathway and pediatric $\mathrm{T}$ acute lymphoblastic leukemia-review. Zhongguo Shi Yan Xue Ye Xue Za Zhi 24: 1269-1274, 2016 (In Chinese).

35. Kong J, Ren G, Jia N, Wang Y, Zhang H, Zhang W, Chen B and Cao Y: Effects of nicorandil in neuroprotective activation of PI3K/AKT pathways in a cellular model of Alzheimer's disease. Eur Neurol 70: 233-241, 2013.

36. Kitagishi Y, Nakanishi A, Ogura Y and Matsuda S: Dietary regulation of PI3K/AKT/GSK-3 $\beta$ pathway in Alzheimer's disease. Alzheimers Res Ther 6: 35, 2014.

37. Li H, Kang T, Qi B, Kong L, Jiao Y, Cao Y, Zhang J and Yang J: Neuroprotective effects of ginseng protein on PI3K/Akt signaling pathway in the hippocampus of $\mathrm{D}$-galactose/ $\mathrm{AlCl} 3$ inducing rats model of Alzheimer's disease. J Ethnopharmacol 179: 162-169, 2016.

38. Zhang B, Wang Y, Li H, Xiong R, Zhao Z, Chu X, Li Q, Sun S and Chen S: Neuroprotective effects of salidroside through PI3K/Akt pathway activation in Alzheimer's disease models. Drug Des Devel Ther 10: 1335-1343, 2016.

39. Ferencik M, Novak M, Rovensky J and Rybar I: Alzheimer's disease, inflammation and non-steroidal anti-inflammatory drugs. Bratisl Lek Listy 102: 123-132, 2001.

40. McGeer EG and McGeer PL: Chronic inflammation in Alzheimer's disease offers therapeutic opportunities. Expert Rev Neurother 1: 53-60, 2001.

41. Quinn J, Montine T, Morrow J, Woodward WR, Kulhanek D and Eckenstein F: Inflammation and cerebral amyloidosis are disconnected in an animal model of Alzheimer's disease. J Neuroimmunol 137: 32-41, 2003.

42. Verdile G, Keane KN, Cruzat VF, Medic S, Sabale M, Rowles J, Wijesekara N, Martins RN, Fraser PE and Newsholme P: Inflammation and oxidative stress: The molecular connectivity between insulin resistance, obesity, and alzheimer's disease. Mediators Inflamm 2015: 105828, 2015.

43. Jiang L, Tang Y and Huang X: Brain cell apoptosis after cerebral hypoxia-ischemia in neonatal rat. Zhonghua YI XUE ZA ZHI 78: 567-569, 1998 (In Chinese).

44. Zhang XQ, Zhang ZM, Yin XL, Zhang K, Cai H and Ling F. Exploring the optimal operation time for patients with hypertensive intracerebral hemorrhage: Tracking the expression and progress of cell apoptosis of prehematomal brain tissues. Chin Med J (Engl) 123: 1246-1250, 2010.

45. Johnson S, Tazik S, Lu D, Johnson C, Youdim MB, Wang J, Rajkowska $\mathrm{G}$ and $\mathrm{Ou} \mathrm{XM}$ : The new inhibitor of monoamine oxidase, M30, has a Neuroprotective effect against dexamethasone-induced brain cell apoptosis. Front Neurosci 4: 180, 2010.

46. Yang S, Zhou G, Liu H, Zhang B, Li J, Cui R and Du Y: Protective effects of p38 MAPK inhibitor SB202190 against hippocampal apoptosis and spatial learning and memory deficits in a rat model of vascular dementia. Biomed Res Int 2013: 215798, 2013.

47. Yürüker V, Nazıroğlu M and Senol N: Reduction in traumatic brain injury-induced oxidative stress, apoptosis, and calcium entry in rat hippocampus by melatonin: Possible involvement of TRPM2 channels. Metab Brain Dis 30: 223-231, 2015.

48. Chatterjee A, Chattopadhyay D and Chakrabarti G: MiR-16 targets Bcl-2 in paclitaxel-resistant lung cancer cells and overexpression of miR-16 along with miR-17 causes unprecedented sensitivity by simultaneously modulating autophagy and apoptosis. Cell Signal 27: 189-203, 2015. 